\title{
Vivencias vitales significativas, bienestar psicológico y resiliencia de estudiantes de alto rendimiento escolar pertenecientes a sectores vulnerables. Un estudio comparativo ${ }^{1}$
}

\author{
Mario Morales², Eduardo Guzmán U. \\ Universidad de SANTIAgo de Chile \\ RECIBIDO 05/08/2016, ACEPTADO 21/10/2016
}

Significant life experience, psychological well-being, and resilience in high performance students from high vulnerability contexts. A comparative study

\begin{abstract}
RESUMEN
Se presentan los resultados de un estudio cuyo objetivo fue conocer la forma en que un grupo de estudiantes de cuarto medio, con alto rendimiento escolar, proveniente de liceos con un índice de vulnerabilidad elevado, (sobre $80 \%$ ), ha vivido diversos sucesos vitales normativos y no normativos en comparación con estudiantes de bajo rendimiento escolar. La población fue de 502 estudiantes pertenecientes a diversos liceos de la Región Metropolitana. Los instrumentos utilizados fueron: Bienestar Psicológico (BIEP-J), Escala de Resiliencia (SVRES) y Escala de Vivencia Vitales Significativas para adolescentes (VVS). Se encontraron diferencias significativas en la satisfacción con el liceo y solicitud de apoyo para la resolución de problemas. En bienestar psicológico, las diferencias fueron en las dimensiones proyectos y control y en resiliencia en los factores de metas, proyectos, autoeficacia y aprendizaje.
\end{abstract}

Palabras clave: Sucesos vitales, rendimiento académico, vulnerabilidad social, adolescencia.

\begin{abstract}
The results are presented of a study comparing high performance High school students with an index of high vulnerability (about 80\%) and their normative and non-normative life events, compared with another group of students with poor school performance. The population included 502 students from various schools in the metropolitan area of Chile. The instruments were used: psychological well-being, (BIEP-J), Resilience Scale (SV-RES); and normative and non-normative life events (VVS). Significant differences were found in satisfaction with the school and support application for problem solving; Regarding their psychological well-being differences occurred in the projects and control factors, and the resilience scale in the factors of goals, project, self-efficacy and learning.
\end{abstract}

Keywords: Life events, school performance, social vulnerability adolescence.

1 Investigación financiada por la Dirección de Investigación y Tecnología (Dicyt), Universidad de Santiago de Chile.

2 E-mail: mario.morales@usach.cl 


\section{INTRODUCCIÓN}

La adolescencia constituye un momento crítico en la formación de la identidad de los seres humanos. Dado el carácter transicional de esta etapa, que ha permitido consolidar una visión particular de esta respecto a los nuevos roles sociales que se corresponden con ella y que deberán cumplir los adolescentes, se ha asociado a profundos cambios en diversas áreas del desarrollo, dada la experimentación de complejidades por medio de crisis y conflictos psicológicos en juego, y que se manifiestan producto de múltiples variables que se generan a partir de distintas dimensiones: física, cognitiva, psicológica y social (Oliva, 2003; Craig y Baucum, 2009).

Desde un punto de vista cognitivo, Jean Piaget planteó que los adolescentes experimentan una transición desde un tipo de pensamiento concreto a un pensamiento formal y abstracto, permitiéndoles formular una serie de críticas acerca de valores familiares, sociales, morales y emocionales. Estas críticas abren oportunidades para comprender, valorar y aceptar o discrepar de los roles y vivencias del mundo cuando estas son o les parecen ilógicas o incongruentes (Craig y Baucum, 2009). Lo anterior permite la interacción del individuo con la sociedad en términos críticos, desarrollando así también una elección de señales y modelos que permitan construir un sentido de identidad personal por medio de un proceso de moratoria psicosocial, es decir, un retraso en la adquisición de responsabilidades sociales aceptadas en ciclos vitales posteriores (González, 2011).

Uno de los conceptos fundamentales dentro de este estudio son los sucesos vitales; estos son entendidos como acontecimientos o sucesos relevantes de las personas y que se desarrollan en determinados momentos de la vida, como por ejemplo, la adolescencia. Tenemos entonces que la pérdida de personas significativas, las buenas o malas experiencias escolares, los distintos cambios socioculturales, etc., resultan vivencias de importancia y relevancia para los adolescentes que las experimentan, pudiendo ser reconocidas como importantes o psicológicamente sanas dentro del proceso de desarrollo evolutivo; o bien pueden alterar el ajuste psicológico y el bienestar personal físico y psicosocial cuando se interpretan como inadecuadas respecto de la no suficiente madurez para afrontarlas (Casullo, 1998).

Estas permanentes vivencias transformadoras a las cuales se alude en la adolescencia, y que han sido estudiadas ampliamente por la psicología del desarrollo y otras áreas afines, suelen sumar complejidad cuando se asocian a distintos factores de riesgo del contexto al cual se pertenece, sean estos socioculturales o económicamente deprivados, aumentando las posibilidades de vulnerabilidad debido a la gran cantidad de elementos estresantes propios de estas condiciones, y por tanto generando la mayor presencia de sucesos vitales no normativos y el impacto que esto produciría en el bienestar psicológico de los jóvenes (Rodríguez, 2002).

Autores como Shulman y Collins (1993), Olivares, Rosa y Olivares (2007), Amutio \& Smith (2008), Lorence, Jiménez y Sánchez (2009), consideran que los 
adolescentes están más expuestos a situaciones de vida generadoras de ansiedad, y estas determinan diversos procesos adaptativos, algunos más adecuados y otros que pueden ser considerados críticos.

No obstante, el hecho de que estos acontecimientos vitales no sean fáciles de prever permite observar una constante fuente de tensión que perturba y modifica la secuencia y el ritmo del ciclo vital de las personas. Parece necesario entonces dar énfasis a la evaluación de las consecuencias de estos sucesos vitales, dado que las experiencias pueden generar en ciertas ocasiones un proceso de fortalecimiento, mientras que en otras pueden desencadenar una serie de consecuencias negativas para el bienestar personal de los adolescentes. Según Lara, Navarro y Navarrete (2004), estas consecuencias pueden ser mediatas o inmediatas, y ambas deben ser consideradas en los procesos de investigación y evaluación de estos fenómenos, dado que aun cuando, desde lo inmediato, el impacto puede ser evaluado por el sujeto como muy negativo, algunas experiencias pueden fortalecer el yo en el largo plazo, mientras que en otras ocasiones debilitarlo.

Cardoso y Alderete (2009) indicaron que jóvenes expuestos a circunstancias adversas manifestaron una inadecuada adaptación social. Sin embargo, también pudieron observar que existían algunos sujetos que, a pesar de estas situaciones adversas, fueron capaces de desarrollar competencias sociales y obtener una adaptación positiva a los distintos contextos. Estas investigaciones concluyen que el proceso de adaptación se relaciona con recursos internos del individuo, así como con recursos externos provenientes desde el soporte social; ambos factores considerados como buenos predictores de la resiliencia, entendida como un proceso dinámico donde las influencias del ambiente como los recursos internos interactúan, permitiendo una adecuación positiva frente a las situaciones adversas, así como una conducta adaptativa ante las situaciones de riesgo (Fullana, 1998; Green \& Conrad, 2002).

Adicionalmente, los mismos autores anteriores indican que los eventos no normativos más estresantes son aquellos relacionados al plano escolar, a situaciones de inadaptación, cambios de clases, repetición de curso y cambios de colegio. Por otra parte, otros estudios indican que constituyen situaciones de vivencia adversas a la experiencia de los jóvenes el plano familiar, los cambios en el sistema familiar, las relaciones entre sus miembros y los modelos de funcionamiento (Cardoso y Alderete, 2009; Ruano y Serra (2001).

Lorence, Jiménez y Sánchez (2009) evaluaron la frecuencia y el impacto emocional de los acontecimientos vitales estresantes en una muestra de 216 adolescentes, encontrando la existencia de una media de por lo menos 8 eventos estresantes en los últimos cinco años, generando todos ellos un alto impacto emocional en los jóvenes. Los eventos más estresantes correspondían al ámbito escolar, mientras que los vividos con mayor intensidad eran aquellos que se relacionaban con el ámbito familiar y personal.

El segundo constructo abordado es el Bienestar Psicológico, el cual se enmarca dentro de la psicología social positiva; este se relaciona con la subjetividad personal 
(Diener, Suh, Lucas y Smith, 1999) y centra su interés en el estudio del desarrollo individual, las experiencias positivas, el bienestar subjetivo o nivel de felicidad, y el funcionamiento óptimo de las personas, las comunidades y la sociedad (Duckworth, Steen \& Seligman, 2005). Es un concepto cuyas definiciones se podrían caracterizar a partir de tres elementos: el carácter subjetivo -que descansa sobre la propia experiencia de la persona-; su dimensión global -que incluye la valoración del sujeto en todas las áreas de su vida-; y la apreciación positiva, ya que su naturaleza va más allá de la mera ausencia de factores negativos (Diener, 1994).

De acuerdo con la psicología positiva, los mayores niveles de bienestar subjetivo suponen colectivamente un mayor desarrollo económico, una cultura en la que las personas tienen una mayor libertad para elegir sobre su propia vida, una preocupación por la igualdad social y la legitimación de relaciones sociales igualitarias (Lima y Novo, 2006; Bilbao, 2008). Por el contrario, menores niveles de bienestar subjetivo se relacionarían con mayor número de problemas de salud mental, de integración social, bajo autoconcepto, entre otros.

Actualmente, uno de los trabajos más replicados en este ámbito es el realizado por Carol Ryff (1989, 1998), el cual plantea una estructura de seis factores propios del bienestar (autoaceptación, dominio del ambiente, sentimiento de realización en la vida, compromiso vital, autonomía y vínculos), obtenidos mediante análisis factoriales exploratorios y confirmatorios en diversas versiones de distinta longitud de las escalas de Ryff (Ryff, 1989; Ryff y Keyes, 1995; Diener et al., 1999; Keyes, Shmotkin y Ryff, 2002). En muestras holandesas, Van Dierendonck (2004) describió que el mejor modelo es el de seis factores con un factor de segundo orden, a pesar de que no todos los ajustes fueron satisfactorios y exigieron la eliminación de ítems (Véliz, 2012). Posteriormente, Díaz et al. (2006) probaron diversos modelos de adaptación de la escala en población española, buscando la estructura más aceptable en relación con el modelo de Ryff.

Salanova, Martínez, Bresó, Llorens y Grau (2005) encontraron relaciones significativas entre bienestar psicológico y rendimiento académico en jóvenes universitarios, señalando que aquellos que poseen mejores calificaciones experimentan menor agotamiento, mayor eficacia en los estudios, mayor dedicación, satisfacción y felicidad. Por su parte, Velásquez et al. (2008), en un estudio descriptivo-correlacional en una universidad de Perú, obtuvieron resultados similares en relación con la existencia de relaciones significativas entre las mismas variables investigadas por Salanova y sus colaboradores. En este contexto, Morales y González (2014) indicaron la importancia de destacar que la sensación subjetiva producto de la experiencia pudiese estar afectada en los estudiantes vulnerables que se han incorporado a la universidad, dada la tensión permanente que experimentan, lo que influiría en su bienestar psicológico.

Finalmente, el último factor a considerar y desarrollar en este estudio es el concepto de resiliencia, constructo que durante los últimos años ha generado múltiples investigaciones, las cuales no están ajenas a diversas controversias con el uso de 
este. Saavedra (2012) señala que las grandes críticas se relacionan con: 1) la ambigüedad y variaciones en la definición del concepto; 2) variaciones en el funcionamiento psicológico entre áreas y entre situaciones de riesgo en sujetos resilientes; 3) la inestabilidad en el fenómeno de resiliencia; y por último 4) la controversia de carácter teórico que coloca en duda la utilidad científica del concepto.

En general, los autores han definido a la resiliencia como una capacidad de adaptación y éxito a pesar de la presencia de factores adversos o de riesgo (Fullan, 1998). Implica enfrentar y desafiar las circunstancias adversas con el propósito de disminuir o superar sus efectos nocivos; es la capacidad del individuo para enfrentarse a las adversidades de la vida, aprender de ellas y salir fortalecido (Grotberg, 2006).

Diversos estudios han considerado múltiples contextos y variables que se relacionarían con el concepto: la resiliencia como concepto multidimensional (Werner, 1995); factores predictores principales de la resiliencia (Masten y Obradovic, 2006); factores protectores que permiten reducir la probabilidad de un resultado negativo en situaciones adversas, atributos personales y del contexto (Grotberg, 2006); e implicaciones de políticas sociales para la intervención basada en el constructo de resiliencia (Luthar y Cichetti, 2000). Específicamente, Finn y Rock (1997) hablan de la resiliencia educacional para referirse a alumnos pertenecientes a minorías raciales de escasos recursos que presentan calificaciones en un nivel aceptable y logran completar la educación escolar.

La consideración de esta variable se respalda en el supuesto que aquellos estudiantes de enseñanza media de sectores de alta vulnerabilidad que presentan mejor rendimiento escolar serían aquellos que han podido enfrentar y superar con éxito una serie de obstáculos provenientes desde el contexto sociocultural en el cual se desenvuelven. Por lo cual, desde esta opción, el concepto de resiliencia refiere a la superación de los múltiples obstáculos dados principalmente por la situación socioeconómica, sociocultural y escolar, adaptándose exitosamente a las tareas que la sociedad les asigna en su etapa del ciclo vital, pero en un ambiente adverso y de vulneración.

El estudio que a continuación se presenta tuvo como objetivo conocer la forma en que un grupo de alumnos de cuarto medio de alto rendimiento escolar, provenientes de liceos con alto índice de vulnerabilidad, vivieron diversos sucesos vitales normativos y no normativos. Al mismo tiempo, se realizó una comparación con estudiantes que poseían bajo rendimiento escolar. Los resultados fueron relacionados con las variables de bienestar psicológico y resiliencia.

\section{MÉTODO}

El estudio se enmarcó dentro de un paradigma metodológico cuantitativo, utilizando un diseño no experimental, descriptivo correlacional y transeccional. La población fue de 502 estudiantes del nivel escolar cuarto medio, provenientes de instituciones escolares con un alto índice de vulnerabilidad escolar (IVE, cercano a un $80 \%$ ) del programa liceos prioritarios del Ministerio de Educación de Chile. Se 
seleccionaron dos grupos; el primero de ellos estuvo constituido por 89 estudiantes con bajo rendimiento académico (RB), situándose a una desviación estándar bajo la media del grupo en los cuatro años de escolaridad inmediatos (primero a cuarto medio). El segundo grupo estuvo constituido por 90 estudiantes de alto rendimiento académico (RA), que se situaron a una o más desviaciones estándar sobre el promedio del curso durante su permanencia en la enseñanza media.

Para la recolección de datos se aplicaron tres instrumentos: El primero fue la escala de bienestar psicológico (BIEP-J), adaptada por Casullo y Castro (2000), que consta de 13 reactivos cuyas puntuaciones van de 1 (en desacuerdo) hasta 3 (de acuerdo). Las dimensiones que evalúa son control, vínculos, proyectos de vida, aceptación. El instrumento posee un nivel de confiabilidad aceptable de 0,69 (alfa de Cronbach) y una adecuada validez, habiendo sido utilizado en otras investigaciones relacionadas en estudios con adolescentes.

El segundo instrumento utilizado fue la escala de resiliencia (SV-RES) para jóvenes y adultos (Saavedra y Villalta, 2008), la cual se basa teóricamente en los modelos del constructo de resiliencia de los autores Grotberg (1996) y Saavedra (2003). Fue validada estadísticamente el año 2007, probándose en población urbana chilena entre 15 y 65 años, y pudiendo ser aplicada individual y colectivamente, e incluso pudiendo ser autoadministrada. Cuenta con 60 ítems con cinco alternativas en escala Likert, que van desde "muy de acuerdo" a "muy en desacuerdo". Permite obtener un puntaje global de resiliencia y un puntaje en las siguientes áreas: identidad, autonomía, satisfacción, pragmatismo, vínculos, redes, modelos, metas, afectividad, autoeficacia, aprendizaje y generatividad. El nivel de confiabilidad de la escala es de 0,74 (alfa de Cronbach).

El afrontamiento de los sucesos vitales normativos y no normativos fue evaluado por la escala VVS en la adolescencia. Este instrumento fue elaborado para fines investigativos por Espinoza, Ferreira y Saballa (2006), teniendo como referencia el discurso vivencial de los adolescentes adquirido a través de técnicas focus group. Los reactivos que constituyen el instrumento definitivo están divididos en las siguientes áreas: personal, sexualidad, salud, escolar, familiar y social. Cada una de estas áreas consta de reactivos que deben ser respondidos por el examinado de acuerdo con los siguientes criterios: "lo viví positivamente", "lo viví negativamente", "lo viví indiferente" y "no lo viví". El instrumento posee un estudio de confiabilidad aceptable para utilizarlo en esta investigación (0.62 en alfa de Cronbach).

Con el propósito de entender mejor las vivencias vitales significativas que acontecen a los adolescentes y que se consideran como normativas, entendiéndose por ello sucesos que forman parte del desarrollo normal, lo que los hace hasta cierto punto, predecibles (Espinosa, Pereira y Saballa, 2006); en el área escolar, se consideran algunas vivencias que se relacionan con obtención de reconocimiento de profesores y logro académico. Estas son experiencias que pueden favorecer la satisfacción personal, otorgando un optimismo frente a otras situaciones escolares negativas. En el área familiar, los adolescentes vivencian de manera negativa la muerte de algunos de los abuelos, y de manera positiva que la madre haya empezado a trabajar fuera del hogar. 
Conflictos entre los padres o con los padres son sucesos que se expresan como negativos. En el área de la sexualidad se aprecian conductas que se extienden desde el autoerotismo, la masturbación, la heterosexualidad, parejas estables, exploraciones sexuales, hasta haber tenido relaciones sexuales por primera vez. Dentro de los sucesos negativos se encuentran las desilusiones amorosas y término de relaciones de pareja. En el área social, se viven como experiencias positivas las manifestaciones masivas o actividades públicas, reencuentro con amistades, y en general relaciones sociales donde se encuentren implicados grupos de pares. En el área de la salud, se aprecia que los adolescentes vivencian de manera positiva conductas que pueden ser factores de riesgo para la salud, como fumar, beber alcohol y probar una droga. Las tendencias de estos comportamientos pueden ser explicadas como el proceso de búsqueda de la identidad y aceptación social (lealtad respecto al grupo). En el área personal, son experimentadas y valoradas como positivas la práctica de algún deporte, la realización de un voluntariado, tener logros e ir configurando su propio estilo de socialización.

En cuanto a los eventos no normativos, estos sucesos en general presentan una baja frecuencia. Son sucesos que pueden aparecer en forma inesperada e impredecible, influyendo e impactando al adolescente. En el área escolar nos encontramos con conductas de fugas, ir en contra de la autoridad, realizar actos arriesgados, etc. En el área familiar, son percibidos como negativos la separación de los padres, la pérdida de trabajo, el vivir con familiares con adicción, las enfermedades graves o sufrir algún accidente. En el área social, son sucesos que se relacionan con la presencia de amigos cercanos que tienen o hayan tenido una enfermedad o accidente grave, y que hayan empeorado las relaciones de amistad. En el área de la salud, los jóvenes viven como negativo haber tenido algún accidente grave, el consumo habitual de alcohol y los cambios de peso. En el área personal viven como positivo haber tenido que trabajar y recibir una remuneración; como suceso negativo es frecuente también pensar en el suicidio.

Se consultó a los adolescentes sobre el nivel de satisfacción que poseían acerca de familia, liceos y amigos. Al mismo tiempo, se consultó sobre las aproximaciones que ellos realizan cuando tienen problemas: profesores, padres, amigos, otros.

Finalmente, se procedió a evaluar la normalidad de los datos por medio de la prueba Kolmogorov-Smirnov (K-S), de forma de decidir el uso de prueba $t$-Student o U. de Mann-Whitney según corresponda. Los resultados indicaron que todas las variables medidas no presentaban una distribución normal, por lo que se decidió utilizar pruebas no paramétricas

\section{RESULTADOS}

Se procedieron a calcular los promedios correspondientes a ambos grupos a comparar según los objetivos descritos. El cuadro $\mathrm{N}^{\circ} 1$ provee de estas medias y las respectivas desviaciones estándar de cada una de las variables analizadas. El promedio del grupo estudiado es de 6,12 ( $\mathrm{N}=90)$; este se calculó considerando 
el rendimiento de todas las asignaturas entre primero y cuarto medio. Al mismo tiempo, el promedio del grupo de rendimiento bajo (R.B.) fue de 4,29 (N=89).

Cuadro $N^{\circ}$ 1. Promedio de notas

\begin{tabular}{cccc}
\hline Grupos & $\mathrm{N}$ & Promedios & d.e. \\
\hline R.B. & 89 & 4.29 & 0.32 \\
R.A. & 90 & 6.12 & 1.96 \\
\hline
\end{tabular}

Con respecto al grado de satisfacción que los estudiantes tienen de su familia, liceo, amigos se muestra en el cuadro siguiente:

Cuadro $\mathrm{N}^{\circ}$ 2. Grado de satisfacción con su familia

\begin{tabular}{ccccccc}
\hline Grupos & & M.S. & S. & P.S. & INS. & Totales \\
\hline R.B. & F & 38 & 24 & 15 & 12 & 89 \\
(89) & $\%$ & 42.5 & 27.3 & 16.5 & 13.7 & 100 \\
R.A. & F & 50 & 22 & 12 & 6 & 90 \\
(90) & $\%$ & 55.3 & 24.8 & 13.4 & 6.4 & 100 \\
\hline
\end{tabular}

M.S. Muy satisfecho, S. Satisfecho, P.S. Poco satisfecho, INS. Insatisfecho

El cuadro $\mathrm{N}^{\mathrm{o}} 2$ nos muestra el grado de satisfacción que tienen los estudiantes respecto a su familia de origen. Se puede apreciar que el $69.8 \%$ de los estudiantes de rendimiento bajo se encuentran satisfechos, al igual que un $80 \%$ del grupo de rendimiento alto. Las diferencias entre los grupos no son significativas.

Cuadro No 3. Grado de satisfacción con su liceo

\begin{tabular}{ccccccc}
\hline Grupos & & M.S. & S. & P.S. & INS. & Totales \\
\hline R.B. & F & 7 & 38 & 30 & 14 & 89 \\
(89) & $\%$ & 8.2 & 42.3 & 33.7 & 15.7 & 100 \\
R.A. & F & 56 & 25 & 5 & 4 & 90 \\
(90) & $\%$ & 62.5 & 27.3 & 5.6 & 4.6 & 100 \\
\hline
\end{tabular}

En el cuadro anterior ( $\left.\mathrm{N}^{\circ} 3\right)$, se aprecian algunas diferencias entre ambos grupos respecto al grado de satisfacción con su liceo. Un $89.8 \%$ del grupo de rendimiento alto se ubica entre muy satisfecho y satisfecho con su liceo, mientras que el grupo de rendimiento bajo es cercano a un $50 \%$. Respecto a la insatisfacción con su liceo, los porcentajes son bajos en los estudiantes de rendimiento alto. Se observan diferencias entre los grupos $\left(\mathrm{Chi}^{2}=62,1, \mathrm{~g} / 1.3, \alpha .001\right)$ 
Cuadro $\mathrm{N}^{\mathrm{o}}$ 4. Grado de satisfacción con sus amigos

\begin{tabular}{ccccccc}
\hline Grupos & & M.S. & S. & P.S. & INS. & Totales \\
\hline R.B. & F & 37 & 38 & 8 & 6 & 89 \\
(89) & $\%$ & 41.1 & 43.5 & 8.7 & 6.6 & 100 \\
R.A. & F & 39 & 34 & 5 & 12 & 90 \\
(90) & $\%$ & 43.2 & 38.3 & 5.3 & 13.2 & 100 \\
\hline
\end{tabular}

En el cuadro $\mathrm{N}^{\mathrm{o}} 4$ se aprecia la diferencia que existe respecto a la satisfacción con los amigos. Se evidencia que no hay diferencias entre ambos grupos; en general los datos son muy cercanos.

Cuadro $\mathrm{N}^{\circ}$ 5. Solicitud de apoyo frente a los conflictos

\begin{tabular}{cccccccc}
\hline Grupos & & A nadie & $\begin{array}{c}\text { Grupo } \\
\text { Amigos }\end{array}$ & Padres & Prof. & Otros & Totales \\
\hline R.B. & F & 36 & 39 & 8 & 2 & 4 & 89 \\
$(89)$ & $\%$ & 40.4 & 43.5 & 8.5 & 2.3 & 5.2 & 100 \\
R.A. & F & 25 & 18 & 30 & 16 & 1 & 90 \\
$(90)$ & $\%$ & 27.8 & 19.5 & 33.5 & 17.9 & 1.3 & 100 \\
\hline
\end{tabular}

En el cuadro $\mathrm{N}^{\circ} 5$ se pueden observar diferencias respecto al apoyo que ellos solicitan frente a la resolución de los conflictos. Mientras que el grupo de estudiantes de rendimiento bajo no recurre a otras personas, y por otro lado un porcentaje importante acude a los amigos; el grupo de alumnos de Rendimiento Alto acuden a los padres y profesores. Las diferencias son significativas $\left(\mathrm{Chi}^{2}=\right.$ $35.18, \mathrm{~g} / 1.3, \alpha .001)$.

Con respecto al Bienestar psicológico, en el siguiente cuadro ( $\left.\mathrm{N}^{\mathrm{o}} 6\right)$ se pueden apreciar los resultados de ambos grupos:

Cuadro $\mathrm{N}^{\mathrm{o}}$ 6. Bienestar Psicológico

\begin{tabular}{ccccccc}
\hline Grupos & & BIEPS & Acept. & Proyec & Vínculos & Control \\
\hline R.B. & Media & 33.36 & 7.33 & 7.75 & 7.91 & 9.37 \\
(89) & d.e & 3.97 & 1.88 & 1.23 & 1.15 & 1.75 \\
R.A. & Media & 34.18 & 7.40 & $8.47^{*}$ & 7.93 & 10.38 \\
(90) & d.e & 2.84 & 1.35 & 1.00 & 1.09 & 1.53 \\
\hline
\end{tabular}

$\mathrm{Z}-3.19 \mathrm{p}=.001) \quad \mathrm{Z} 3,25 \mathrm{p}=.04$

Se evidencian algunas diferencias en la Escala de Bienestar Psicológico. En la escala total, los resultados son similares. En la dimensión proyección, las diferencias son significativas $(Z=3.19, p=0.01)$ entre estudiantes de alto y de bajo rendimiento (U. de Mann-Whitney). Se puede observar que los estudiantes de rendimiento alto 
logran identificar la construcción de metas y proyectos; se evidencia un sentido y un valor por la vida, dotan de significado a las actividades que realizan y poseen noción y visión de futuro. El segundo factor en que se observan diferencias es en la dimensión Control de Situaciones $(\mathrm{Z}=3.25, \mathrm{p}=0.04)$, donde existe una moderada sensación de control y auto competencia. Los jóvenes se perciben con ciertas herramientas para crear o manipular contextos para adecuarlos a sus propias necesidades e intereses.

Respecto a la Escala de Resiliencia, los resultados se pueden observar en la siguiente tabla.

Cuadro $N^{\circ}$ 7. Escala de Resiliencia

\begin{tabular}{|c|c|c|c|c|c|c|}
\hline Grupos & & Identidad & Autonomía & Satisfacción & Pragmatismo & Vínculos \\
\hline $\begin{array}{l}\text { R.B. } \\
\text { (89) }\end{array}$ & $\begin{array}{c}\text { Media } \\
\text { d.e }\end{array}$ & $\begin{array}{c}62.1 \\
21.56\end{array}$ & $\begin{array}{c}59.29 \\
25.2\end{array}$ & $\begin{array}{c}49.54 \\
27.3\end{array}$ & $\begin{array}{l}65.54 \\
23.78\end{array}$ & $\begin{array}{l}56.61 \\
26.40\end{array}$ \\
\hline $\begin{array}{l}\text { R.A } \\
(90)\end{array}$ & $\begin{array}{c}\text { Media } \\
\text { d.e } \\
\text { W } \\
\end{array}$ & $\begin{array}{c}59.58 \\
27.7 \\
0.75 \text { n.s. }\end{array}$ & $\begin{array}{c}61.8 \\
27.4 \\
0.19 \text { n.s } \\
\end{array}$ & $\begin{array}{c}46.14 \\
26.17 \\
4.25 \text { n.s. }\end{array}$ & $\begin{array}{c}55.02 \\
27.7 \\
2.17 \mathrm{n.s} \\
\end{array}$ & $\begin{array}{c}52.71 \\
29.4 \\
\text { 0.27 n.s. }\end{array}$ \\
\hline Grupos & & Redes & Modelos & Metas & Afectividad & Aprendizaje \\
\hline $\begin{array}{l}\text { R.B. } \\
\text { (89) }\end{array}$ & $\begin{array}{c}\text { Media } \\
\text { d.e }\end{array}$ & $\begin{array}{l}53.9 \\
21.8\end{array}$ & $\begin{array}{c}52.08 \\
23.3\end{array}$ & $\begin{array}{l}53.4 \\
22.3\end{array}$ & $\begin{array}{l}66.5 \\
24.7\end{array}$ & $\begin{array}{l}54.1 \\
21.7\end{array}$ \\
\hline \multirow[t]{4}{*}{$\begin{array}{l}\text { R.A. } \\
(90)\end{array}$} & $\begin{array}{c}\text { Media } \\
\text { d.e } \\
\text { W }\end{array}$ & $\begin{array}{c}57.26 \\
19.27 \\
3.27 \text { n.s. }\end{array}$ & $\begin{array}{c}63.12 \\
22.9 \\
.032 \mathrm{~s} .\end{array}$ & $\begin{array}{l}66.4 \\
25.3 \\
.043 \mathrm{~s}\end{array}$ & $\begin{array}{c}51.7 \\
29.0 \\
2.89 \text { n.s. }\end{array}$ & $\begin{array}{l}67.4 \\
26.5 \\
.05 \mathrm{~s} .\end{array}$ \\
\hline & \multicolumn{2}{|c|}{ Grupos } & \multicolumn{2}{|c|}{ Autoeficacia } & Generatividad & \\
\hline & $\begin{array}{l}\text { R.B. } \\
(89)\end{array}$ & $\begin{array}{c}\text { Media } \\
\text { d.e }\end{array}$ & $\begin{array}{l}50 . \\
25 .\end{array}$ & & $\begin{array}{c}70.1 \\
17.46\end{array}$ & \\
\hline & $\begin{array}{l}\text { R.A. } \\
(90)\end{array}$ & $\begin{array}{c}\text { Media } \\
\text { d.e } \\
\text { W }\end{array}$ & $\begin{array}{r}66 . \\
29 . \\
0,03\end{array}$ & & $\begin{array}{c}64.6 \\
23.1 \\
5,27 \text { n.s. }\end{array}$ & \\
\hline
\end{tabular}

Se puede apreciar respecto a las diferentes dimensiones de la escala de Resiliencia, que en los factores donde se evidencian diferencias entre ambos grupos, y que favorecen a los estudiantes de rendimiento alto, son: modelos que tiene relación con juicios que consideran el papel de las redes sociales cercanas para apoyar la superación de situaciones que son problemáticas; metas que se refieren a juicios que considera el valor de contexto de metas y redes sociales que están por encima de la situación problemática; autoeficacia, relacionada con juicio sobre las posibilidades de éxito que el sujeto reconoce en sí mismo ante una situación problemática; y aprendizaje, que refiere a juicios que logran valorar una situación problemática como una posibilidad de aprendizaje. 
El estudio de correlaciones entre la Escala de Bienestar Psicológico y de Resiliencia arroja los siguientes resultados: Control e identidad (.31**); Control y autoeficacia $\left(.30^{* *}\right)$; Control y generatividad (.29**); Vínculos y afectividad (.29**), Proyecto y autonomía $\left(.25^{*}\right)$; Autocontrol y pragmatismo $\left(.28^{*}\right)$.

Con relación a los resultados que arroja la Escala de Vivencias que han tenido los jóvenes de rendimiento alto y rendimiento bajo frente a los sucesos vitales normativos, se puede apreciar que en el ámbito escolar las diferencias se expresan en tres ítems del área: cambio de colegio, logro académico y el reconocimiento habitual de algún profesor.

Cuadro $\mathrm{N}^{\circ}$ 8. Cambio en el colegio

\begin{tabular}{ccccccc}
\hline Grupos & & No lo viví & Indif. & Positiv. & Negativ. & Total. \\
\hline R.B. & f & 49 & 10 & 17 & 13 & 88 \\
(89) & $\%$ & 55.7 & 11.3 & 19.3 & 14.7 & 98,8 \\
R.A. & f & 37 & 7 & 37 & 8 & 89 \\
(90) & $\%$ & 41.1 & 8.0 & 41.1 & 9.1 & 98.8 \\
\hline
\end{tabular}

Respecto a este ítem, las diferencias entre ambos grupos arrojan un $\mathrm{Chi}^{2}$ de 8.31, g/l., 3 significativo al .040. Se observa que un gran número de estudiantes de Rendimiento bajo que no han vivido este suceso, es bajo el porcentaje de los estudiantes que lo han vivido negativamente; sin embargo, la diferencia se evidencia en aquellos alumnos que lo han vivido positivamente, siendo mayor en el grupo de estudiantes de rendimiento alto.

Cuadro $\mathrm{N}^{\circ}$ 9. Logro académico

\begin{tabular}{ccccccc}
\hline Grupos & & No lo viví & Indif. & Positiv. & Negativ. & Total. \\
\hline R.B. & f & 54 & 8 & 24 & 3 & 89 \\
(89) & $\%$ & 60.5 & 8.9 & 26.8 & 3.8 & 100 \\
R.A. & f & 41 & 5 & 43 & 1 & 90 \\
(90) & $\%$ & 46.1 & 5.5 & 48.2 & 1.1 & 100 \\
\hline
\end{tabular}

Con relación al logro académico obtenido por los alumnos (Cuadro $\mathrm{N}^{\circ}$ 9), los resultados indican diferencias significativas entre ambos grupos, obteniéndose un $\mathrm{Chi}^{2}$ de 11.17, g/1. 3, $\alpha$.011. Las diferencias se observan en aquellos estudiantes de alto rendimiento que han vivido positivamente los logros académicos experimentados en el colegio. Se evidencia además que existe un alto porcentaje de estudiantes de rendimiento bajo que no han vivido este suceso escolar. 
Cuadro $\mathrm{N}^{\mathrm{o}} 10$. Reconocimiento habitual de algún profesor

\begin{tabular}{ccccccc}
\hline Grupos & & No lo viví & Indif. & Positiv. & Negativ. & Total. \\
\hline R.B. & f & 41 & 13 & 24 & 11 & 89 \\
(89) & $\%$ & 46.1 & 14,6 & 26,9 & 12.3 & 100 \\
R.A. & f & 37 & 1 & 46 & 6 & 90 \\
(90) & $\%$ & 41.1 & 1.1 & 51.1 & 6.6 & 100 \\
\hline
\end{tabular}

Ante el reconocimiento habitual que pueden tener los profesores frente al alumno, se observa una relación significativa entre las variables, con un valor $\mathrm{Chi}^{2}$ de 11.9, g/l. 3, significativo al .008. El porcentaje de estudiantes de rendimiento alto que han vivido positivamente este evento es alto con respecto a los estudiantes de rendimiento bajo; en las demás categorías se aprecian algunas diferencias en la cantidad de estudiantes de rendimiento bajo que han vivido de manera indiferentes el suceso escolar.

En el ámbito familiar no se encontraron diferencias significativas. Los reactivos se relacionaron con la pérdida de los abuelos, conflictos con los padres y trabajo de la madre fuera de casa.

En el ámbito de la sexualidad se puede apreciar lo siguiente:

Cuadro $\mathrm{N}^{\circ} 11$. Haber tenido voluntariamente relaciones sexuales por vez primera

\begin{tabular}{ccccccc}
\hline Grupos & & No lo viví & Indif. & Positiv. & Negativ. & Total. \\
\hline R.B. & f & 27 & 2 & 59.5 & 7 & 89 \\
(89) & $\%$ & 30.3 & 2.24 & 57.7 & 7.86 & 100 \\
R.A. & $\mathrm{f}$ & 48 & 0 & 41 & 1 & 90 \\
(90) & $\%$ & 53.3 & 0 & 45.5 & 1.11 & 100 \\
\hline
\end{tabular}

Respecto a la experiencia de haber tenido relaciones sexuales por primera vez, se puede visualizar que en el grupo de rendimiento alto más de la mitad de los jóvenes refieren no haber vivido la experiencia; mientras que en el grupo de rendimiento bajo cerca de un $70 \%$ la han vivido, y la valoran como algo positivo. En general, los demás valores se encuentran muy cercanos entre ambos grupos. El reactivo aparece significativo con $\mathrm{Chi}^{2}=9.29, \mathrm{~g} / \mathrm{l} .3, \alpha .026$.

Cuadro $\mathrm{N}^{\circ} 12$. Uso de un anticonceptivo

\begin{tabular}{ccccccc}
\hline Grupos & & No lo viví & Indif. & Positiv. & Negativ. & Total. \\
\hline R.B. & f & 32 & 20 & 16 & 21 & 89 \\
(89) & $\%$ & 35.9 & 22.4 & 17.9 & 23.6 & 100 \\
R.A. & F & 53 & 22 & 9 & 6 & 90 \\
(90) & $\%$ & 58.8 & 24.4 & 10 & 6.7 & 100 \\
\hline
\end{tabular}


Las diferencias en este ítem solo se dan en primer lugar en los porcentajes de no haber vivido esta experiencia. Un $35.9 \%$ no la han vivido y de aquellos que la han vivido, un $23.6 \%$ la ha valorado de manera negativa. En los estudiantes de rendimiento alto se evidencia una vivencia baja tanto negativa como positiva frente al reactivo. Los valores son significativos, obteniéndose un $\mathrm{Chi}^{2} \mathrm{de}$ 7.84, g/l. 3 significativo al 049 .

En el área social de la escala, los resultados indican que no hubo diferencias entre los grupos. Los reactivos tienen que ver con participación en actividades masivas (marchas, tomas), reencuentro con amigos y la asunción del rol de líder.

En el área de la salud, tampoco se evidencia diferencias entre ambos grupos. Los reactivos tienen que ver con haber tenido alguna experiencia con drogas, alcohol y tabaco. No se evidencian diferencias significativas.

En el área Personal, se observa diferencia en el ítem asociado al fracaso que pudo haber tenido en áreas relacionadas con el deporte o las artes (Cuadro $\mathrm{N}^{\circ} 13$ ).

Cuadro $\mathrm{N}^{\mathrm{o}} 13$. He tenido fracasos deportivos y artísticos

\begin{tabular}{ccccccc}
\hline Grupos & & No lo viví & Indif. & Positiv. & Negativ. & Total. \\
\hline R. B. & f & 58 & 4 & 12 & 15 & 89 \\
(89) & $\%$ & 65.2 & 4.5 & 13.5 & 16.8 & 98.8 \\
R.A. & f & 70 & 9 & 1 & 10 & 90 \\
(90) & $\%$ & 77.7 & 10.0 & 1.1 & 11.1 & 100 \\
\hline
\end{tabular}

Se evidencia que un porcentaje alto de estudiantes de rendimiento alto no han vivido el suceso, y dentro de los que sí reconocen haberlo vivido, un 10\% lo han valorado negativamente. Mientras, en el grupo de alumnos con rendimiento bajo un $62.5 \%$ argumenta no haberlo vivido. En aquellos que han tenido la experiencia, la reacción ha sido tanto positiva como negativa. Las diferencias son significativas, lo que arroja un $\mathrm{Chi}^{2}$ 10.59, g/l. 3 significativo al .032.

Con respecto a los eventos no normativos, se puede apreciar que las diferencias se dan en algunos ítems relacionados con el área escolar, familiar y salud.

El área escolar, dentro de los eventos no normativos, evidencia diferencias en algunos reactivos relacionados con la frecuencia de las sanciones escolares.

Cuadro $\mathrm{N}^{\mathrm{0}}$ 14. Recibo sanción escolar al menos una vez por semana.

\begin{tabular}{ccccccc}
\hline Grupos & & No lo viví & Indif. & Positiv. & Negativ. & Total. \\
\hline R. B. & f & 55 & 12 & 7 & 15 & 89 \\
(89) & $\%$ & 61.8 & 13.48 & 7.86 & 16.9 & 100 \\
R.A. & f & 85 & 3 & 1 & 1 & 90 \\
(90) & $\%$ & 94.4 & 3.3 & 1,1 & 1.1 & 100 \\
\hline
\end{tabular}


Con relación a la frecuencia de haber recibido sanción al menos una vez por semana, se puede apreciar que existen diferencias significativas entre ambos grupos $\left(\mathrm{Chi}^{2}\right.$ $15.0 ; \mathrm{g} / 1.3 ; .02$.). De los estudiantes de Rendimiento Bajo, un 38\% han vivido esta experiencia, mientras que solo un 5.8 de los alumnos de Rendimiento Alto han experimentado la sanción. De aquellos alumnos de Rendimiento Bajo que han tenido esta experiencia, un $16.9 \%$ la han vivido de manera negativa.

En el área familiar, las diferencias se evidencian en la convivencia del estudiante al vivir con algún familiar que es adicto a la droga. Los resultados se pueden observar en el siguiente cuadro.

Cuadro $\mathrm{N}^{\mathrm{o}} 15$. Vivo con un familiar que tiene o ha tenido algún tipo de adicción (drogas, alcohol).

\begin{tabular}{ccccccc}
\hline Grupos & & No lo viví & Indif. & Positiv. & Negativ. & Total. \\
\hline R. B. & f & 52 & 10 & 5 & 22 & 89 \\
(89) & $\%$ & 58.4 & 11,2 & 5.6 & 24.7 & 100 \\
R.A. & f & 62 & 15 & 2 & 11 & 90 \\
(90) & $\%$ & 68.8 & 16.6 & 2.22 & 12.22 & 100 \\
\hline
\end{tabular}

Más de la mitad de los estudiantes de ambos grupos indican no haber vivido la experiencia. Sin embargo, en el grupo de Rendimiento Bajo, hay un porcentaje mayor que en el grupo de Rendimiento Alto respecto a haber vivido el suceso de manera negativa. Las diferencias son significativas $\left(\mathrm{Chi}^{2}=7.85, \mathrm{~g} / \mathrm{l} .3, \alpha .049\right)$.

Otro reactivo en que también se observan diferencias es la ocurrencia de vivir con un familiar que ha tenido o que tiene una enfermedad o accidente grave.

Cuadro $\mathrm{N}^{\mathrm{o}} 16$. Vivo con un familiar que ha tenido o que tiene una enfermedad o accidente grave

\begin{tabular}{ccccccc}
\hline Grupos & & No lo viví & Indif. & Positiv. & Negativ. & Total. \\
\hline R. B. & F & 50 & 9 & 21 & 9 & 89 \\
(89) & $\%$ & 56.17 & 10.11 & 23.5 & 10.11 & 100 \\
R.A. & F & 75 & 7 & 4 & 4 & 90 \\
(90) & $\%$ & 83.3 & 7.77 & 4.44 & 4.44 & 100 \\
\hline
\end{tabular}

El maltrato físico o psicológico al interior de las familias (Cuadro $\left.\mathrm{N}^{\mathrm{o}} 17\right)$ se da en un porcentaje muy bajo en el grupo de rendimiento alto, se reporta que solo lo han vivido un 3,4\%, mientras que en los estudiantes de Rendimiento Bajo el porcentaje es de un $25.9 \%$. Un $16.8 \%$ han vivido esta experiencia de manera negativa. Se observan diferencias significativas entre ambos grupos. $\left(\mathrm{Chi}^{2}=7,40, \mathrm{~g} / 13, \alpha .05\right)$. 
Cuadro $\mathrm{N}^{\mathrm{o}}$ 17. En mi familia ocurre maltrato físico o psicológico.

\begin{tabular}{ccccccc}
\hline Grupos & & No lo viví & Indif. & Positiv. & Negativ. & Total. \\
\hline R. B. & F & 66 & 4 & 4 & 15 & 89 \\
(89) & $\%$ & 74.1 & 4.49 & 4.49 & 16.8 & 100 \\
R.A. & F & 87 & 1 & 1 & 1 & 90 \\
(90) & $\%$ & 96.6 & 1.1 & 1.1 & 1.1 & 100 \\
\hline
\end{tabular}

En el Área de la Salud, se puede apreciar que las diferencias se establecen en la ocurrencia de haber experimentado algún accidente grave (Cuadro $\mathrm{N}^{\circ} 18$ ).

Cuadro 18. He tenido algún accidente grave

\begin{tabular}{ccccccc}
\hline & & No lo viví & Indif. & Positiv. & Negativ. & Total. \\
\hline R. B & f & 48 & 9 & 23 & 9 & 89 \\
(89) & $\%$ & 53.9 & 10.1 & 25.8 & 10.11 & 100 \\
R.A. & f & 61 & 17 & 10 & 2 & 90 \\
(90) & $\%$ & 67.7 & 18.8 & 11.1 & 2.22 & 100 \\
\hline
\end{tabular}

Se aprecian diferencias entre los estudiantes de Rendimiento Alto y de Rendimiento Bajo. Un porcentaje alto no han vivido esta experiencia, observándose una valoración positiva de la experiencia en un $25 \%$ de quienes la han vivido. ( $\mathrm{Chi}^{2} \mathrm{de}$ $8.22, \mathrm{~g} / 1.3, \alpha .042$.

En las áreas personal, sexualidad y social, no se evidenciaron diferencias significativas en ninguno de los reactivos de la escala.

Otra de las comparaciones que se realizaron fue la cantidad de eventos normativos y no normativos ocurridos en ambos grupos. Los resultados indican que no se evidencian diferencias significativas entre ellos.

\section{DISCUSIÓN}

El objetivo del estudio fue comparar dos grupos de estudiantes de sectores con altos índices de vulnerabilidad, quienes presentaban distintos rendimiento escolares en variables relacionadas con el bienestar psicológico, resiliencia y la forma de enfrentar los sucesos vitales normativos y no normativos que han tenido que vivir.

En primer lugar, los estudiantes se diferenciaron por el rendimiento escolar, variable que permitió constituir dos grupos de estudiantes: aquellos que han alcanzado alto rendimiento escolar (promedio de notas 6.1) en los cuatro años de enseñanza media, y aquellos alumnos que presentaron rendimiento bajo (promedio de notas 4,3). 
Frente a la satisfacción de ambos grupos de estudiantes con la familia, liceo y amigos, las diferencias aparecen respecto al liceo o institución donde estudian. Los estudiantes de rendimiento alto se sienten más satisfechos con su liceo que el grupo de rendimiento bajo. Las relaciones con sus profesores, el refuerzo permanente que ellos reciben por parte de la dirección y de los maestros, y el rendimiento mismo, generan un grado de satisfacción mayor que el grupo de comparación. Respecto a la satisfacción con la familia, a pesar de que hay un mayor reconocimiento de esta en el grupo de rendimiento alto, esta no es significativa. Resultado similar se evidencia en la satisfacción con los amigos, evento en que no aparecen diferencias significativas. No obstante, es importante destacar que ambos grupos expresan un alto grado de satisfacción.

Otro estudio realizado por Chavarría y Barra (2014) da cuenta de que en una muestra de 358 adolescentes de ambos sexos con edades comprendidas entre los 14 y 19 años, los niveles de satisfacción vital en las dimensiones amigos y familia son altos. En cambio, se encontraron menores niveles de satisfacción en las dimensiones colegio y entorno, en comparación con las otras tres variables.

Frente al apoyo que expresan ambos grupos respecto a los conflictos que deben enfrentar, las diferencias son importantes. Mientras que el grupo de rendimiento bajo recurre a los amigos, y un gran porcentaje no recurre a ninguna persona; los estudiantes de rendimiento alto señalan que los padres y especialmente los profesores son a quienes más recurren. Es importante destacar la adecuada relación que existe entre los estudiantes de rendimiento alto con sus profesores.

Los resultados indicaron que en la Escala de Bienestar Psicológico las dimensiones que diferenciaron a ambos grupos de estudiantes fueron: Proyección y Control. La primera se refiere a las capacidades que ellos poseen para establecer proyectos y metas, e intentar darle sentido y significado a la vida. La segunda dimensión implica una moderada sensación de control y auto competencia. En general, los jóvenes se perciben con ciertas herramientas para crear o manipular contextos para luego adecuarlos a sus propias necesidades e intereses. Con respecto a las demás dimensiones, en general todas ellas aparecen como un bienestar psicológico calificado moderado.

En relación a los vínculos psicosociales, se percibe que los jóvenes poseen la capacidad para relacionarse con las demás personas, intentando evitar el aislamiento, estableciendo vínculos y compromisos con los demás, y quizás en ocasiones experimentando frustraciones. Por su parte, la aceptación de sí mismo, también se encuentra en la categoría de moderada, dado que intenta aceptar múltiples aspectos sobre sí mismo, positivos y negativos, así como aquellos que responden de su pasado, aunque en determinadas circunstancias experimentan algunas desilusiones e insatisfacciones consigo mismos. Sin embargo, en estas dimensiones no hubo diferencias significativas.

Otro resultado importante a destacar se aprecia en las variables de bienestar psicológico, dado que al asociarlas con el rendimiento escolar, no aparecen resultados significativos. Esto se contradice con los resultados de otros estudios como los de 
Chávez (2006), quien encontró una correlación alta en jóvenes universitarios entre el bienestar psicológico (en todas sus dimensiones) y el rendimiento académico. Por su parte, Velásquez et al. (2008), en una muestra en jóvenes universitarios, obtuvieron resultados que señalaron que existe relación entre el bienestar psicológico y el rendimiento. Además, los investigadores concluyen que se observó, tanto en hombres y mujeres, como en las diferentes facultades, que la relación era significativa en los jóvenes y adultos, pero no aparecía en los adolescentes.

Es importante considerar que el estudio realizado corresponde a un grupo de estudiantes de un contexto escolar con alto índice de vulnerabilidad, mientras que las investigaciones señaladas son muestras de contextos universitarios.

La resiliencia fue otro factor que nos entregó información interesante. La hipótesis sostenida era que aquellos jóvenes que presentaban alto rendimiento escolar perteneciente a liceos vulnerables poseían un mayor nivel de resiliencia para afrontar los sucesos vitales normativos y no normativos que los estudiantes de rendimiento bajo. No obstante, los factores donde aparecen diferencias fueron modelos, metas, aprendizaje y autoeficacia.

El factor modelo se refiere a juicios que se asocian con la convicción del papel de las redes sociales cercanas para apoyar la superación de situaciones problemáticas nuevas. La presencia de estas redes de apoyo permite una reducción de situaciones que pueden provocar estrés. Ambos grupos presentan un alto grado de satisfacción con los amigos. Sin embargo, el grupo de estudiantes de rendimiento alto considera a los profesores como parte importante de la red social.

La presencia de los profesores aparece como un rol activo en las relaciones que establecen los estudiantes como formas de ayuda para resolver sus problemas. Los padres cumplen también un rol importante respecto a las formas de abordar las situaciones problemáticas. No obstante, un grupo de estudiantes (de ambos grupos) frente a las situaciones problemáticas no acuden a nadie.

Las metas son juicios que tienen relación al valor contextual de metas y redes sociales por sobre la situación problemática. Existe en el grupo de estudiantes de rendimiento alto un puntaje más alto que en el grupo de estudiantes de rendimiento bajo. En los primeros, sus metas tienen que ver con continuar con estudios superiores, llegar a ser profesional. Esto se fundamenta con los resultados de otro estudio donde se revela que las motivaciones de los estudiantes para continuar con sus estudios responde al compromiso de ayudar económicamente a sus familias, superar las necesidades económicas que tanto tiempo han permanecido en ellos y alcanzar un desarrollo personal al transformarse en profesionales (Morales y Sepúlveda, en prensa).

El factor aprendizaje también genera diferencias significativas entre ambos grupos. Se trata principalmente en la forma que tienen los sujetos para transformar y valorar una situación problemática como una manera de aprender; en otras palabras, es la capacidad de aprender de los aciertos y errores. Los jóvenes de rendimiento alto han desarrollado mejor esta capacidad que los jóvenes de rendimiento bajo. 
Los estudiantes de rendimiento alto poseen una mayor tendencia de autoeficacia, entendida como la capacidad del sujeto para darse cuenta sobre las posibilidades de éxito que las personas reconocen en sí mismos frente a una situación problemática. La autoeficacia, de acuerdo con Bandura (1995), consideraría tres tipos de expectativas. De la situación: las consecuencias son provocadas por el evento independiente de la acción del sujeto. Del resultado: refiere a la creencia que una conducta producirá determinados resultados. De la Autoeficacia, percibida como la creencia que una persona posee sobre sus capacidades para desempeñar las acciones suficientes para obtener determinados resultados. Un bajo nivel de autoeficacia se asocia a determinados estado de depresión, ansiedad, mientras que un alto nivel de autoeficacia facilita el procesamiento de información y el desempeño cognitivo en distintos contextos, incluyendo la toma de decisiones y el logro académico (Bandura, 1997). Estos resultados concuerdan con el estudio realizado.

Respecto a la escala de los sucesos vitales normativos, se evidencian diferencias significativas en los eventos ocurridos en la escuela como cambios de colegio, logros académicos, reconocimiento de profesores, favoreciendo a los estudiantes de alto rendimiento escolar, quienes han vivido estos sucesos de manera positiva. En el área de la sexualidad se observan diferencias respecto a la experiencia en las relaciones sexuales, en las cuales ambos grupos las han experimentado de manera positiva; sin embargo, la frecuencia ha sido mayor en los estudiantes de bajo rendimiento. Respecto a las áreas familiar, social y personal no se evidencia diferencias significativas entre ambos grupos.

Frente a los eventos no normativos, la diferencia de ambos grupos se aprecia en el área escolar, específicamente en la frecuencia de las sanciones en la escuela; en el plano familiar, la presencia de algún miembro con una enfermedad grave y en la frecuencia de maltrato, siendo esto último mayor en los estudiantes de bajo rendimiento. Respecto al área de la salud, los resultados indican la presencia de algún evento relacionado con un accidente personal, donde los estudiantes de alto rendimiento lo han vivido de forma más positiva que los estudiantes de bajo rendimiento. Respecto al área social y personal, no se evidencian diferencias significativas entre ambos grupos.

Los resultados de este estudio son relevantes, especialmente para configurar una serie de factores protectores y de riesgo que pudiesen interferir en los logros escolares. Los procesos educativos que se realizan en las instituciones escolares deben responder a las necesidades de los estudiantes, deben considerar el conocimiento que se tenga del joven por medio de aspectos relacionados con su bienestar psicológico, componentes de una conducta resiliente, el nivel de satisfacción con los diversos actores que acompañan al adolescente y la forma en que este enfrenta los sucesos vitales tanto normativos como no normativos que vivencia.

Por otra parte, este estudio promueve la generación de bases curriculares que contemplen el desarrollo integral de los estudiantes de enseñanza media, 
asumiendo un carácter procesual y de continuidad, en que se incentive y promueva un acercamiento al adolescente procurando la prevención y promoción de factores psicosociales saludables.

Finalmente, el rol de los psicólogos y orientadores, en virtud de estos resultados, nos entrega diversas pistas que permitan contemplar programas de capacitación a los diversos profesionales en promoción y prevención de salud mental, considerando la perspectiva abordada respecto a factores psicosociales saludables, especialmente en los liceos con altos índices de vulnerabilidad.

\section{CONCLUSIONES}

1. Los estudiantes de rendimiento alto se sienten más satisfechos con su liceo que el grupo de rendimiento bajo.

2. Los resultados indicaron que en la Escala de Bienestar Psicológico las dimensiones que diferenciaron a ambos grupos de estudiantes fueron: Proyección y Control. La primera se refiere a las capacidades que ellos poseen para establecer proyectos y metas, e intentar darle sentido y significado a la vida. La segunda dimensión implica una moderada sensación de control y auto competencia.

3. En la variable Resiliencia se evidenció que las diferencias entre ambos grupos fueron en las dimensiones relacionadas con modelos, metas, aprendizaje y autoeficacia.

4. Frente a los eventos normativos se evidencian diferencias significativas en los eventos ocurridos en la escuela como cambios de colegio, logros académicos, reconocimiento de profesores. En los eventos no normativos, la diferencia de ambos grupos se aprecia en el área escolar, específicamente en la frecuencia de las sanciones en la escuela; en el plano familiar, la presencia de algún miembro con una enfermedad grave y en la frecuencia de maltrato, siendo esto último mayor en los estudiantes de bajo rendimiento.

\section{REFERENCIAS BIBLIOGRÁFICAS}

Amutio, A. \& Smith, J. (2008). Stress and irrational beliefs in college students. Ansiedad Estrés, 14 (2-3), 211-220.

Bandura, A. (1995). Self-Efficacy in changing societies. New York: Cambridge University Press.

Bandura, A. (1997). Self-efficacy: The exercise of Control. New York: Freeman.

Bilbao, M. (2008). Creencias sociales y bienestar: Valores, creencias básicas, impacto de los hechos vitales y crecimiento psicológico. (Tesis Doctoral). Universidad del País Vasco - Euskal Herriko Unibertsitatea, España. Recuperado el 30 de noviembre de 2015 desde http://dspace2.conicyt.cl/handle/10533/89109 
Cardozo, G. y Alderete, A. (2009). Adolescentes en riesgo psicosocial y resiliencia. Psicología desde el Caribe, 23, 148-182. Recuperado el 18 de noviembre de 2015 desde http://rcientificas.uninorte.edu.co/index.php/psicologia/article/viewFile/650/342

Casullo, M. y Castro, A. (2000). Evaluación del Bienestar Psicológico en estudiantes adolescentes argentinos. Revista de Psicología, XVIII (2), 25-49. Recuperado el 15 de diciembre de 2015 desde http://revistas.pucp.edu.pe/ index.php/psicologia/article/view/6840/6974

Casullo, M. (1998). Adolescentes en Riesgo. Identificación y orientación psicológica. Buenos Aires: Paidós.

Chavarría, M. y Barra, E. (2014). Satisfacción vital en adolescentes: Relación con la autoeficacia y el apoyo social percibido. Terapia Psicológica, 32 (1), 4146. Recuperado el 30 de octubre de 2015 desde http://www.scielo.cl/pdf/ terpsicol/v32n1/art04.pdf

Craig, G. y Baucum, D (2009). Desarrollo psicológico. México: Pearson educación.

Díaz, D., Rodríguez, R., Blanco, A., Moreno, B., Gallardo, I., Valle, C. y Van Dierendonck, D. (2006). Adaptación española de las Escalas de Bienestar Psicológico de Ryff. Psicothema, 18 (3), 572-577. Recuperado el 30 de octubre de 2015 desde http://www.psicothema.com/pdf/3255.pdf

Diener, E. (1994). Assessing subjective well-being: Progress and opportunities. Social indicators research, 31, 103-157. doi=10.1007/BF01207052.

Diener, E., Suh, E. Lucas, R. y Smith, H. (1999). Subjective well-being. Three decades of progress. Psychological bulletin, 125 (2), 276-302. doi=10.1037/00332909.125.2.276.

Duckworth, A., Steen, T. y Seligman, M. (2005). Positive psychology in clinical practice. Annual Review of Clinical Psychology, 1, 629-651. doi=10.1146/ annurev.clinpsy.1.102803.144154.

Espinoza, M., Pereira, M. y Saballa D. (2006). Vivencias vitales significativas y bienestar psicológico; Reorientación escolar como factor protector. Tesis para optar al título de Psicólogo. Mención Psicología Educacional e infanto juvenil. Universidad de Santiago de Chile.

Finn, J. y Rock, D. (1997). Academic success among students at risk for school failure. Journal of Applied Psychology, 82, 221-234. Recuperado el 20 de diciembre de 2015 desde http://www.viriya.net/jabref/Academic_success_ among_students_at_risk_for_school_failure.pdf

Fullana, J. (1998). La búsqueda de factores del fracaso escolar. Revista de investigación educativa, 16 (1), 47-70. Recuperado el 18 de diciembre de 2015 desde http://revistas.um.es/rie/article/view/122411/115031 
González, M. (2011). Identidad, un proceso constante, dinámico y fluido. Revista universitaria de investigación y diálogo académico, 7 (3), 19-28. Recuperado el 3 de enero de 2016 desde http://conhisremi.iuttol.edu.ve/pdf/ ARTI000131.pdf

Green, R., \& Conrad, A. (2002). Basic assumptions and terms. En R. Green (Ed). Resiliency. An Integrated Approach to practice, Policy and Research (pp. 29-62). Washington DC: National Association of Social Workers Press.

Grotberg, E. (2006). ¿Qué entendemos por resiliencia?, ¿cómo promoverla?, ¿cómo utilizarla?. Barcelona: Gedisa.

Grotberg, E. (1996). A Guide to Promoting Resilience in Children: strengthening the spirit human. La Haya: Bernard Van Leer Foundation.

Keyes, C., Shmotkin, D. y Ryff, C. (2002) Optimizing well-being: The empirical encounter of two traditions. Journal of Personality and Social Psychology, 82 (6), 1007-1022. doi= http://dx.doi.org/10.1037/0022-3514.82.6.1007

Lara, M., Navarro, C. y Navarrete, L. (2004). Influencia de los sucesos vitales y el apoyo social en una intervención psicoeducativa para mujeres con depresión. Salud Pública de México, 46 (5), 378-387. Recuperado el 5 de enero de 2016 desde http://www.scielo.org.mx/pdf/spm/v46n5/a03v46n5.pdf

Lima, M. L. y Novo, R. (2006). Nós por cá todos bem? Bem-estar subjetivo e social em Portugal ena Europa. En J. Vala y A. Torres (Eds.), Contextos e attitudes sociais na Europa (pp. 147-182). Lisboa: Instituto de Ciências Sociais.

Lorence, B., Jiménez, L. y Sánchez, J. (2009). Un análisis de los sucesos vitales estresantes experimentados por adolescentes que crecen en familias usuarias de los servicios sociales comunitarios. Revista Portularia. IX (1), 115-126. Recuperado el 6 de enero de 2016 desde http://www.redalyc.org/ pdf/1610/161013161011.pdf

Luthar, S., y Cicchetti, D. (2000). The construct of resilience: Implications for interventions and social policies. Development and Psychopathology, 12 (4), 857-88. Recuperado el 10 de octubre de 2015 desde http://www.ncbi.nlm. nih.gov/pmc/articles/PMC1903337/

Masten, A. y Obradovic, J. (2006). Competence and resilience in development. Annals of the New York Academy of Sciences, 1094 (1), 13-27. Recuperado el 6 de enero de 2016 desde http://www.ncbi.nlm.nih.gov/pubmed/17347338

Morales, M. y González, A. (2014). Resiliencia-autoestima-bienestar psicológico y capacidad intelectual de estudiantes de cuarto medio de buen rendimiento de liceos vulnerables. Estudios pedagógicos, XI (1), 215-228. Recuperado el 05 de enero de 2015 desde http://www.scielo.cl/scielo.php?script=sci arttext\&pid=S0718-07052014000100013\&lng=es\&tlng=es. $10.4067 /$ S0718-07052014000100013 
Morales, M. y Sepúlveda M. (2015). Alto rendimiento académico en estudiantes de enseñanza media de liceos vulnerables. Facultad de Humanidades. Universidad de Santiago de Chile. .

Oliva, A. (2003). Adolescencia en España a principios del siglo XXI. Cultura y Educación, 15 (4), 373-383. Recuperado el 6 de enero de 2016 desde http:// personal.us.es/oliva/siglo\%2021.pdf

Olivares-Olivares, P., Rosa-Alcázar, A. y Olivares-Rodríguez, J. (2007). Validez social de la intervención en adolescentes con fobia social: padres frente a profesores. Terapia psicológica, 25 (1), 63-71. doi= http://dx.doi.org/10.4067/ S0718-48082007000100005

Rodríguez, P. (2002) Análisis de historias clínicas de estudiantes universitarios con dificultades económicas. (Tesis de especialización en clínica comunitaria). Universidad Católica Andrés Bello. Caracas, Venezuela.

Ruano, R., y Serra, E. (2001). Sucesos vitales y tensiones en familias con hijos adolescentes: Life events and strains in families with teenagers. Estudios pedagógicos, 27, 55-64. Recuperado el 12 de enero de 2015 desde http:// mingaonline.uach.cl/pdf/estped/n27/art04.pdf

Ryff, C. y Keyes, C. (1995). The structure of psychological well-being revisited. Journal of Personality and Social Psychology, 69 (4), 719-727. doi= http:// dx.doi.org/10.1037/0022-3514.69.4.719.

Ryff, C. (1989). Happiness is everything: or is it? Explorations on the meaning of psychological well-being. Journal of Personality and Social Psychology, 57 (6), 1069-1081. http://dx.doi.org/10.1037/0022-3514.57.6.1069

Saavedra, E. (2012).Una aproximación al estado del arte de la investigación en resiliencia. En J. Catalán. (Ed.). Investigación orientada al cambio en Psicología Educacional (pp. 167-182). La Serena, Universidad de La Serena.

Saavedra, E. y Villalta, M. (2008). Escala de Resiliencia SV-RES. Para jóvenes y adultos (2 ${ }^{\mathrm{a}}$ edición). Santiago de Chile: CEANIM.

Saavedra, E. (2003). El Enfoque Cognitivo Procesal Sistémico, como posibilidad de Intervenir educativamente en la formación de sujetos Resilientes. España: Universidad de Valladolid.

Salanova, M., Martínez, I., Bresó E., Llorens S. y Grau R. (2005). Bienestar psicológico en estudiantes universitarios: facilitadores y obstaculizadores del desempeño académico. Anales de psicología, 21 (1), 170-180. Recuperado el 12 de octubre de 2015 desde http://revistas.um.es/analesps/article/ view/27261/26451

Shulman, S. y Collins, W. (Ed.) (1993). Father-adolescent relationships. San Francisco: Jossey-Bass. 
Van Dierendonck, D. (2004). The construct validity of Ryff's Scale of Psychological well-being and its extension with spiritual well-being. Personality and Individual Differences, 36(3), 629-644. Recuperado el 18 de diciembre de 2015 desde http://www.sciencedirect.com/science/article/pii/ S0191886903001223

Velásquez C., Montgomery, W., Montero, V., Pomalaya, R., Dioses, A., Velásquez, N., Araki, R. y Reynoso, D. (2008). Bienestar psicológico, asertividad y rendimiento académico en estudiantes universitarios Sanmarquinos. Revista de Investigación en Psicología, 11 (2), 139-152. Recuperado el 18 de diciembre de 2015 desde http://revistasinvestigacion.unmsm.edu.pe/index. php/psico/article/view/3845/3078

Véliz, A. (2012). Propiedades psicométricas de la escala de bienestar psicológico y su estructura factorial en universitarios chilenos. Psicoperspectivas. Individuo y sociedad, 11 (2), 143-163. doi: 10.5027/ psicoperspectivas -Vol 11-Issue2-fulltext-196.

Werner, E. (1995). Resilience in development. Current directions in psychological science, 4 (3), 81-85. doi $=10.1111 / 1467-8721 . e p 10772327$. 